\title{
Weighted boundedness of multilinear operators associated to singular integral operators with non-smooth kernels
}

\section{Daqing Lu*}

\section{"Correspondence:} Idqcslgdx@163.com College of Mathematics, Changsha University of Science and Technology, Changsha, 410077 P.R. China

\begin{abstract}
In this paper, we establish the weighted sharp maximal function inequalities for a multilinear operator associated to a singular integral operator with non-smooth kernel. As an application, we obtain the boundedness of the operator on weighted Lebesgue and Morrey spaces.
\end{abstract}

MSC: 42B20; 42B25

Keywords: multilinear operator; singular integral operator; sharp maximal function; weighted $B M O$; weighted Lipschitz function

\section{Introduction and preliminaries}

As the development of singular integral operators (see $[1,2]$ ), their commutators and multilinear operators have been well studied. In [3-5], the authors prove that the commutators generated by the singular integral operators and $B M O$ functions are bounded on $L^{p}\left(R^{n}\right)$ for $1<p<\infty$. Chanillo (see [6]) proves a similar result when the singular integral operators are replaced by the fractional integral operators. In [7, 8], the boundedness for the commutators generated by the singular integral operators and Lipschitz functions on Triebel-Lizorkin and $L^{p}\left(R^{n}\right)(1<p<\infty)$ spaces is obtained. In $[9,10]$, the boundedness for the commutators generated by the singular integral operators and the weighted $B M O$ and Lipschitz functions on $L^{p}\left(R^{n}\right)(1<p<\infty)$ spaces is obtained (also see [11]). In [12,13], some singular integral operators with non-smooth kernels are introduced, and the boundedness for the operators and their commutators is obtained (see [14-17]). Motivated by these, in this paper, we study multilinear operators generated by singular integral operators with non-smooth kernels and the weighted Lipschitz and BMO functions.

In this paper, we study some singular integral operators as follows (see [13]).

Definition 1 A family of operators $D_{t}, t>0$, is said to be an 'approximation to the identity' if, for every $t>0, D_{t}$ can be represented by a kernel $a_{t}(x, y)$ in the following sense:

$$
D_{t}(f)(x)=\int_{R^{n}} a_{t}(x, y) f(y) d y
$$

for every $f \in L^{p}\left(R^{n}\right)$ with $p \geq 1$, and $a_{t}(x, y)$ satisfies

$$
\left|a_{t}(x, y)\right| \leq h_{t}(x, y)=C t^{-n / 2} \rho\left(|x-y|^{2} / t\right)
$$

C 2014 Lu; licensee Springer. This is an Open Access article distributed under the terms of the Creative Commons Attribution License (http://creativecommons.org/licenses/by/2.0), which permits unrestricted use, distribution, and reproduction in any medium, provided the original work is properly cited. 
where $\rho$ is a positive, bounded and decreasing function satisfying

$$
\lim _{r \rightarrow \infty} r^{n+\epsilon} \rho\left(r^{2}\right)=0
$$

for some $\epsilon>0$.

Definition 2 A linear operator $T$ is called a singular integral operator with non-smooth kernel if $T$ is bounded on $L^{2}\left(R^{n}\right)$ and associated with the kernel $K(x, y)$ so that

$$
T(f)(x)=\int_{R^{n}} K(x, y) f(y) d y
$$

for every continuous function $f$ with compact support, and for almost all $x$ not in the support of $f$.

(1) There exists an 'approximation to the identity' $\left\{B_{t}, t>0\right\}$ such that $T B_{t}$ has the associated kernel $k_{t}(x, y)$ and there exist $c_{1}, c_{2}>0$ so that

$$
\int_{|x-y|>c_{1} t^{1 / 2}}\left|K(x, y)-k_{t}(x, y)\right| d x \leq c_{2} \quad \text { for all } y \in R^{n} .
$$

(2) There exists an 'approximation to the identity' $\left\{A_{t}, t>0\right\}$ such that $A_{t} T$ has the associated kernel $K_{t}(x, y)$ which satisfies

$$
\left|K_{t}(x, y)\right| \leq c_{4} t^{-n / 2} \quad \text { if }|x-y| \leq c_{3} t^{1 / 2}
$$

and

$$
\left|K(x, y)-K_{t}(x, y)\right| \leq c_{4} t^{\delta / 2}|x-y|^{-n-\delta} \quad \text { if }|x-y| \geq c_{3} t^{1 / 2}
$$

for some $\delta>0, c_{3}, c_{4}>0$. Moreover, let $m$ be a positive integer and $b$ be a function on $R^{n}$. Set

$$
R_{m+1}(b ; x, y)=b(x)-\sum_{|\alpha| \leq m} \frac{1}{\alpha !} D^{\alpha} b(y)(x-y)^{\alpha} .
$$

The multilinear operator related to the operator $T$ is defined by

$$
T^{b}(f)(x)=\int_{R^{n}} \frac{R_{m+1}(b ; x, y)}{|x-y|^{m}} K(x, y) f(y) d y .
$$

Note that the commutator $[b, T](f)=b T(f)-T(b f)$ is a particular operator of the multilinear operator $T^{b}$ if $m=0$. The multilinear operator $T^{b}$ is a non-trivial generalization of the commutator. It is well known that commutators and multilinear operators are of great interest in harmonic analysis and have been widely studied by many authors (see [18-20]). The main purpose of this paper is to prove sharp maximal inequalities for the multilinear operator $T^{b}$. As an application, we obtain the weighted $L^{p}$-norm inequality and Morrey space boundedness for the multilinear operator $T^{b}$. 
Now, let us introduce some notations. Throughout this paper, $Q$ will denote a cube of $R^{n}$ with sides parallel to the axes. For any locally integrable function $f$, the sharp maximal function of $f$ is defined by

$$
M^{\#}(f)(x)=\sup _{Q \ni x} \frac{1}{|Q|} \int_{Q}\left|f(y)-f_{Q}\right| d y,
$$

where, and in what follows, $f_{Q}=|Q|^{-1} \int_{Q} f(x) d x$. It is well known that (see $\left.[1,2]\right)$

$$
M^{\#}(f)(x) \approx \sup _{Q \ni x} \inf _{c \in C} \frac{1}{|Q|} \int_{Q}|f(y)-c| d y .
$$

Let

$$
M(f)(x)=\sup _{Q \ni x} \frac{1}{|Q|} \int_{Q}|f(y)| d y .
$$

For $\eta>0$, let $M_{\eta}^{\#}(f)(x)=M^{\#}\left(|f|^{\eta}\right)^{1 / \eta}(x)$ and $M_{\eta}(f)(x)=M\left(|f|^{\eta}\right)^{1 / \eta}(x)$.

For $0<\eta<n, 1 \leq p<\infty$ and the non-negative weight function $w$, set

$$
M_{\eta, p, w}(f)(x)=\sup _{Q \ni x}\left(\frac{1}{w(Q)^{1-p \eta / n}} \int_{Q}|f(y)|^{p} w(y) d y\right)^{1 / p}
$$

We write $M_{\eta, p, w}(f)=M_{p, w}(f)$ if $\eta=0$.

The sharp maximal function $M_{A}(f)$ associated with the 'approximation to the identity' $\left\{A_{t}, t>0\right\}$ is defined by

$$
M_{A}^{\#}(f)(x)=\sup _{x \in Q} \frac{1}{|Q|} \int_{Q}\left|f(y)-A_{t_{Q}}(f)(y)\right| d y,
$$

where $t_{Q}=l(Q)^{2}$ and $l(Q)$ denotes the side length of $Q$. For $\eta>0$, let $M_{A, \eta}^{\#}(f)=M_{A}^{\#}\left(|f|^{\eta}\right)^{1 / \eta}$.

The $A_{p}$ weight is defined by (see [1]), for $1<p<\infty$,

$$
A_{p}=\left\{w \in L_{\text {loc }}^{1}\left(R^{n}\right): \sup _{Q}\left(\frac{1}{|Q|} \int_{Q} w(x) d x\right)\left(\frac{1}{|Q|} \int_{Q} w(x)^{-1 /(p-1)} d x\right)^{p-1}<\infty\right\}
$$

and

$$
A_{1}=\left\{w \in L_{\mathrm{loc}}^{p}\left(R^{n}\right): M(w)(x) \leq C w(x), \text { a.e. }\right\}
$$

Given a non-negative weight function $w$. For $1 \leq p<\infty$, the weighted Lebesgue space $L^{p}\left(R^{n}, w\right)$ is the space of functions $f$ such that

$$
\|f\|_{L^{p}(w)}=\left(\int_{R^{n}}|f(x)|^{p} w(x) d x\right)^{1 / p}<\infty .
$$

For $0<\beta<1$ and the non-negative weight function $w$, the weighted Lipschitz space $\operatorname{Lip}_{\beta}(w)$ is the space of functions $b$ such that

$$
\|b\|_{L_{i p_{\beta}(w)}}=\sup _{Q} \frac{1}{w(Q)^{\beta / n}}\left(\frac{1}{w(Q)} \int_{Q}\left|b(y)-b_{Q}\right|^{p} w(x)^{1-p} d y\right)^{1 / p}<\infty,
$$


and the weighted $B M O$ space $B M O(w)$ is the space of functions $b$ such that

$$
\|b\|_{B M O(w)}=\sup _{Q}\left(\frac{1}{w(Q)} \int_{Q}\left|b(y)-b_{Q}\right|^{p} w(x)^{1-p} d y\right)^{1 / p}<\infty .
$$

Remark (1) It has been known that (see [9, 21]), for $b \in \operatorname{Lip}_{\beta}(w), w \in A_{1}$ and $x \in Q$,

$$
\left|b_{Q}-b_{2^{k} Q}\right| \leq C k\|b\|_{L i p_{\beta}(w)} w(x) w\left(2^{k} Q\right)^{\beta / n} .
$$

(2) It has been known that (see $[1,21])$, for $b \in B M O(w), w \in A_{1}$ and $x \in Q$,

$$
\left|b_{Q}-b_{2^{k} Q}\right| \leq C k\|b\|_{B M O(w)} w(x) .
$$

(3) Let $b \in \operatorname{Lip}_{\beta}(w)$ or $b \in B M O(w)$ and $w \in A_{1}$. By [22], we know that spaces $\operatorname{Lip}_{\beta}(w)$ or $B M O(w)$ coincide and the norms $\|b\|_{L i p_{\beta}(w)}$ or $\|b\|_{B M O(w)}$ are equivalent with respect to different values $1 \leq p<\infty$.

Definition 3 Let $\varphi$ be a positive, increasing function on $R^{+}$, and let there exist a constant $D>0$ such that

$$
\varphi(2 t) \leq D \varphi(t) \quad \text { for } t \geq 0
$$

Let $w$ be a non-negative weight function on $R^{n}$ and $f$ be a locally integrable function on $R^{n}$. Set, for $0 \leq \eta<n$ and $1 \leq p<n / \eta$,

$$
\|f\|_{L^{p, \eta, \varphi}(w)}=\sup _{x \in R^{n}, d>0}\left(\frac{1}{\varphi(d)^{1-p \eta / n}} \int_{Q(x, d)}|f(y)|^{p} w(y) d y\right)^{1 / p}
$$

where $Q(x, d)=\left\{y \in R^{n}:|x-y|<d\right\}$. The generalized fractional weighted Morrey space is defined by

$$
L^{p, \eta, \varphi}\left(R^{n}, w\right)=\left\{f \in L_{\mathrm{loc}}^{1}\left(R^{n}\right):\|f\|_{L^{p, \eta, \varphi}(w)}<\infty\right\}
$$

We write $L^{p, \eta, \varphi}\left(R^{n}\right)=L^{p, \varphi}\left(R^{n}\right)$ if $\eta=0$, which is the generalized weighted Morrey space. If $\varphi(d)=d^{\delta}, \delta>0$, then $L^{p, \varphi}\left(R^{n}, w\right)=L^{p, \delta}\left(R^{n}, w\right)$, which is the classical Morrey space (see $[23,24])$. If $\varphi(d)=1$, then $L^{p, \varphi}\left(R^{n}, w\right)=L^{p}\left(R^{n}, w\right)$, which is the weighted Lebesgue space (see [1]).

As the Morrey space may be considered as an extension of the Lebesgue space, it is natural and important to study the boundedness of the operator on the Morrey spaces (see [22, 25-27]).

\section{Theorems and lemmas}

We shall prove the following theorems.

Theorem 1 Let $T$ be a singular integral operator with non-smooth kernel as given in Definition $2, w \in A_{1}, 0<\eta<1,1<r<\infty$ and $D^{\alpha} b \in B M O(w)$ for all $\alpha$ with $|\alpha|=m$. Then there 
exists a constant $C>0$ such that, for any $f \in C_{0}^{\infty}\left(R^{n}\right)$ and $\tilde{x} \in R^{n}$,

$$
M_{A, \eta}^{\#}\left(T^{b}(f)\right)(\tilde{x}) \leq C \sum_{|\alpha|=m}\left\|D^{\alpha} b\right\|_{B M O(w)} w(\tilde{x}) M_{r, w}(f)(\tilde{x}) .
$$

Theorem 2 Let $T$ be a singular integral operator with non-smooth kernel as given in Definition $2, w \in A_{1}, 0<\eta<1,1<r<\infty, 0<\beta<1$ and $D^{\alpha} b \in \operatorname{Lip}_{\beta}(w)$ for all $\alpha$ with $|\alpha|=m$. Then there exists a constant $C>0$ such that, for any $f \in C_{0}^{\infty}\left(R^{n}\right)$ and $\tilde{x} \in R^{n}$,

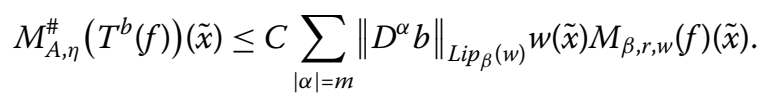

Theorem 3 Let $T$ be a singular integral operator with non-smooth kernel as given in Definition $3, w \in A_{1}, 1<p<\infty$ and $D^{\alpha} b \in B M O(w)$ for all $\alpha$ with $|\alpha|=m$. Then $T^{b}$ is bounded from $L^{p}\left(R^{n}, w\right)$ to $L^{p}\left(R^{n}, w^{1-p}\right)$.

Theorem 4 Let $T$ be a singular integral operator with non-smooth kernel as given in Definition 3, $w \in A_{1}, 1<p<\infty, 0<D<2^{n}$ and $D^{\alpha} b \in B M O(w)$ for all $\alpha$ with $|\alpha|=m$. Then $T^{b}$ is bounded from $L^{p, \varphi}\left(R^{n}, w\right)$ to $L^{p, \varphi}\left(R^{n}, w^{1-p}\right)$.

Theorem 5 Let $T$ be a singular integral operator with non-smooth kernel as given in Definition 3, $w \in A_{1}, 0<\beta<1,1<p<n / \beta, 1 / q=1 / p-\beta / n$ and $D^{\alpha} b \in \operatorname{Lip}_{\beta}(w)$ for all $\alpha$ with $|\alpha|=m$. Then $T^{b}$ is bounded from $L^{p}\left(R^{n}, w\right)$ to $L^{q}\left(R^{n}, w^{1-q}\right)$.

Theorem 6 Let $T$ be a singular integral operator with non-smooth kernel as given in Definition 3, $w \in A_{1}, 0<\beta<1,0<D<2^{n}, 1<p<n / \beta, 1 / q=1 / p-\beta / n$ and $D^{\alpha} b \in \operatorname{Lip}_{\beta}(w)$ for all $\alpha$ with $|\alpha|=m$. Then $T^{b}$ is bounded from $L^{p, \beta, \varphi}\left(R^{n}, w\right)$ to $L^{q, \varphi}\left(R^{n}, w^{1-q}\right)$.

To prove the theorems, we need the following lemmas.

Lemma 1 (see [1, p.485]) Let $0<p<q<\infty$, and for any function $f \geq 0$, we define that, for $1 / r=1 / p-1 / q$,

$$
\|f\|_{W L^{q}}=\sup _{\lambda>0} \lambda\left|\left\{x \in R^{n}: f(x)>\lambda\right\}\right|^{1 / q}, \quad N_{p, q}(f)=\sup _{Q}\left\|f \chi_{Q}\right\|_{L^{p}} /\left\|\chi_{Q}\right\|_{L^{r}}
$$

where the sup is taken for all measurable sets $Q$ with $0<|Q|<\infty$. Then

$$
\|f\|_{W L^{q}} \leq N_{p, q}(f) \leq(q /(q-p))^{1 / p}\|f\|_{W L^{q}}
$$

Lemma 2 (see $[12,13]$ ) Let $T$ be a singular integral operator with non-smooth kernel as given in Definition 2. Then $T$ is bounded on $L^{p}\left(R^{n}, w\right)$ for $w \in A_{p}$ with $1<p<\infty$, and weak $\left(L^{1}, L^{1}\right)$ bounded.

Lemma $3([12,13])$ Let $\left\{A_{t}, t>0\right\}$ be an 'approximation to the identity'. For any $\gamma>0$, there exists a constant $C>0$ independent of $\gamma$ such that

$$
\left|\left\{x \in R^{n}: M(f)(x)>D \lambda, M_{A}^{\#}(f)(x) \leq \gamma \lambda\right\}\right| \leq C \gamma\left|\left\{x \in R^{n}: M(f)(x)>\lambda\right\}\right|
$$


for $\lambda>0$, where $D$ is a fixed constant which only depends on $n$. Thus, for $f \in L^{p}\left(R^{n}\right), 1<p<$ $\infty, 0<\eta<\infty$ and $w \in A_{1}$,

$$
\left\|M_{\eta}(f)\right\|_{L^{p}(w)} \leq C\left\|M_{A, \eta}^{\#}(f)\right\|_{L^{p}(w)} .
$$

Lemma 4 (see $[1,6])$ Let $0 \leq \eta<n, 1 \leq s<p<n / \eta, 1 / q=1 / p-\eta / n$ and $w \in A_{1}$. Then

$$
\left\|M_{\eta, s, w}(f)\right\|_{L^{q(w)}} \leq C\|f\|_{L^{p}(w)}
$$

Lemma 5 (see $[12,13])$ Let $\left\{A_{t}, t>0\right\}$ be an 'approximation to the identity', $0<D<2^{n}$, $1<p<\infty, 0<\eta<\infty, w \in A_{1}$ and $w \in A_{1}$. Then

$$
\left\|M_{\eta}(f)\right\|_{L^{p, \varphi}(w)} \leq C\left\|M_{A, \eta}^{\#}(f)\right\|_{L^{p, \varphi}(w)} .
$$

Lemma 6 (see [22, 25]) Let $0 \leq \eta<n, 0<D<2^{n}, 1 \leq s<p<n / \eta, 1 / q=1 / p-\eta / n$ and $w \in A_{1}$. Then

$$
\left\|M_{\eta, s, w}(f)\right\|_{L^{q, \varphi}(w)} \leq C\|f\|_{L^{p, \eta, \varphi}(w)} .
$$

Lemma 7 (see [19]) Let $b$ be a function on $R^{n}$ and $D^{\alpha} A \in L^{q}\left(R^{n}\right)$ for all $\alpha$ with $|\alpha|=m$ and any $q>n$. Then

$$
\left|R_{m}(b ; x, y)\right| \leq C|x-y|^{m} \sum_{|\alpha|=m}\left(\frac{1}{|\tilde{Q}(x, y)|} \int_{\tilde{Q}(x, y)}\left|D^{\alpha} b(z)\right|^{q} d z\right)^{1 / q},
$$

where $\tilde{Q}$ is the cube centered at $x$ and having side length $5 \sqrt{n}|x-y|$.

Lemma 8 Let $\left\{A_{t}, t>0\right\}$ be an 'approximation to the identity', $w \in A_{1}$ and $b \in B M O(w)$.

Then, for every $f \in L^{p}(w), p>1,1<r<\infty$ and $\tilde{x} \in R^{n}$,

$$
\sup _{Q \ni \tilde{x}} \frac{1}{|Q|} \int_{Q}\left|A_{t_{Q}}\left(\left(b-b_{Q}\right) f\right)(y)\right| d y \leq C\|b\|_{B M O(w)} w(\tilde{x}) M_{r, w}(f)(\tilde{x}),
$$

where $t_{Q}=l(Q)^{2}$ and $l(Q)$ denotes the side length of $Q$.

Proof We write, for any cube $Q$ with $\tilde{x} \in Q$,

$$
\begin{aligned}
& \frac{1}{|Q|} \int_{Q}\left|A_{t_{Q}}\left(\left(b-b_{Q}\right) f\right)(x)\right| d x \\
& \leq \frac{1}{|Q|} \int_{Q} \int_{R^{n}} h_{t_{Q}}(x, y)\left|\left(b(y)-b_{Q}\right) f(y)\right| d y d x \\
& \leq \frac{1}{|Q|} \int_{Q} \int_{Q} h_{t_{Q}}(x, y)\left|\left(b(y)-b_{Q}\right) f(y)\right| d y d x \\
& \quad+\sum_{k=0}^{\infty} \frac{1}{|Q|} \int_{Q} \int_{2^{k+1} Q \backslash 2^{k} Q} h_{t_{Q}}(x, y)\left|\left(b(y)-b_{Q}\right) f(y)\right| d y d x \\
& =I+I I .
\end{aligned}
$$


We have, by Hölder's inequality,

$$
\begin{aligned}
I & \leq \frac{C}{|Q||Q|} \int_{Q} \int_{Q}\left|\left(b(y)-b_{Q}\right) f(y)\right| d y d x \\
& \leq \frac{C}{|Q|} \int_{Q}\left|b(y)-b_{Q}\right| w(y)^{-1 / r}|f(y)| w(y)^{1 / r} d y \\
& \leq \frac{C}{|Q|}\left(\int_{Q}\left|b(y)-b_{Q}\right|^{r^{\prime}} w(y)^{1-r^{\prime}} d y\right)^{1 / r^{\prime}}\left(\int_{Q}|f(y)|^{r} w(y) d y\right)^{1 / r} \\
& \leq \frac{C}{|Q|}\|b\|_{B M O(w)} w(Q)^{1 / r^{\prime}} w(Q)^{1 / r}\left(\frac{1}{w(Q)} \int_{Q}|f(y)|^{r} w(y) d y\right)^{1 / r} \\
& \leq C\|b\|_{B M O(w)} \frac{w(Q)}{|Q|} M_{r, w}(f)(\tilde{x}) \\
& \leq C\|b\|_{B M O(w)} w(\tilde{x}) M_{r, w}(f)(\tilde{x}) .
\end{aligned}
$$

For $I I$, notice for $x \in Q$ and $y \in 2^{k+1} Q \backslash 2^{k} Q$, then $|x-y| \geq 2^{k-1} t_{Q}$ and $h_{t_{Q}}(x, y) \leq C \frac{s\left(2^{2(k-1)}\right)}{|Q|}$, then

$$
\begin{aligned}
& I I \leq C \sum_{k=0}^{\infty} s\left(2^{2(k-1)}\right) \frac{1}{|Q||Q|} \int_{Q} \int_{2^{k+1} Q}\left|\left(b(y)-b_{Q}\right) f(y)\right| d y d x \\
& \leq C \sum_{k=0}^{\infty} 2^{k n} s\left(2^{2(k-1)}\right) \frac{1}{\left|2^{k+1} Q\right|} \\
& \times \int_{2^{k+1} Q}\left|\left(b(y)-b_{2^{k+1} Q}\right)+\left(b_{2^{k+1} Q}-b_{Q}\right)\right||f(y)| d y \\
& \leq C \sum_{k=0}^{\infty} 2^{k n} s\left(2^{2(k-1)}\right)\left|2^{k+1} Q\right|^{-1}\left(\int_{2^{k+1} Q}\left|b(y)-b_{2^{k+1} Q}\right|^{r^{\prime}} w(y)^{1-r^{\prime}} d y\right)^{1 / r^{\prime}} \\
& \times\left(\int_{2^{k+1} Q}|f(y)|^{r} w(y) d y\right)^{1 / r} \\
& +C \sum_{k=0}^{\infty} 2^{k n} s\left(2^{2(k-1)}\right)\left|2^{k+1} Q\right|^{-1} k\|b\|_{B M O(w)} w(\tilde{x})\left(\int_{2^{k+1} Q}|f(y)|^{r} w(y) d y\right)^{1 / r} \\
& \times\left(\frac{1}{\left|2^{k+1} Q\right|} \int_{2^{k+1} Q} w(y)^{-1 /(r-1)} d y\right)^{(r-1) / r} \\
& \times\left(\frac{1}{\left|2^{k+1} Q\right|} \int_{2^{k+1} Q} w(y) d y\right)^{1 / r}\left|2^{k+1} Q\right| w\left(2^{k+1} Q\right)^{-1 / r} \\
& \leq C\|b\|_{B M O(w)} \sum_{k=0}^{\infty} k 2^{k n} s\left(2^{2(k-1)}\right)\left(\frac{w\left(2^{k+1} Q\right)}{\left|2^{k+1} Q\right|}+w(\tilde{x})\right) \\
& \times\left(\frac{1}{w\left(2^{k+1} Q\right)} \int_{2^{k+1} Q}|f(y)|^{r} w(y) d y\right)^{1 / r} \\
& \leq C\|b\|_{B M O(w)} \sum_{k=0}^{\infty} k 2^{k n} s\left(2^{2(k-1)}\right) w(\tilde{x}) M_{r, w}(f)(\tilde{x}) \\
& \leq C\|b\|_{B M O(w)} w(\tilde{x}) M_{r, w}(f)(\tilde{x}),
\end{aligned}
$$


where the last inequality follows from

$$
\sum_{k=1}^{\infty} k 2^{(k-1) n} s\left(2^{2(k-1)}\right) \leq C \sum_{k=1}^{\infty} k 2^{-(k-1) \epsilon}<\infty
$$

for some $\epsilon>0$. This completes the proof.

Lemma 9 Let $\left\{A_{t}, t>0\right\}$ be an 'approximation to the identity', $w \in A_{1}, 0<\beta<1,1<r<\infty$ and $b \in \operatorname{Lip}_{\beta}(w)$. Then, for every $f \in L^{p}(w), p>1$ and $\tilde{x} \in R^{n}$,

$$
\sup _{Q \ni \tilde{x}} \frac{1}{|Q|} \int_{Q}\left|A_{t_{Q}}\left(\left(b-b_{Q}\right) f\right)(y)\right| d y \leq C\|b\|_{L i p_{\beta}(w)} w(\tilde{x}) M_{\beta, w, r}(f)(\tilde{x}) .
$$

The same argument as in the proof of Lemma 8 will give the proof of Lemma 9, we omit the details.

\section{Proofs of theorems}

Proof of Theorem 1 It suffices to prove for $f \in C_{0}^{\infty}\left(R^{n}\right)$ and some constant $C_{0}$ that the following inequality holds:

$$
\left(\frac{1}{|Q|} \int_{Q}\left|T^{b}(f)(x)-A_{t_{Q}}\left(T^{b}(f)\right)(x)\right|^{\eta} d x\right)^{1 / \eta} \leq C \sum_{|\alpha|=m}\left\|D^{\alpha} b\right\|_{B M O(w)} w(\tilde{x}) M_{r, w}(f)(\tilde{x}),
$$

where $t_{Q}=d^{2}$ and $d$ denotes the side length of $Q$. Fix a cube $Q=Q\left(x_{0}, d\right)$ and $\tilde{x} \in Q$. Let $\tilde{Q}=5 \sqrt{n} Q$ and $\tilde{b}(x)=b(x)-\sum_{|\alpha|=m} \frac{1}{\alpha !}\left(D^{\alpha} b\right)_{\tilde{Q}} x^{\alpha}$, then $R_{m}(b ; x, y)=R_{m}(\tilde{b} ; x, y)$ and $D^{\alpha} \tilde{b}=$ $D^{\alpha} b-\left(D^{\alpha} b\right)_{\tilde{Q}}$ for $|\alpha|=m$. We write, for $f_{1}=f \chi_{\tilde{Q}}$ and $f_{2}=f \chi_{R^{n} \backslash \tilde{Q}}$,

$$
\begin{aligned}
T^{b}(f)(x)= & \int_{R^{n}} \frac{R_{m}(\tilde{b} ; x, y)}{|x-y|^{m}} K(x, y) f_{1}(y) d y \\
& -\sum_{|\alpha|=m} \frac{1}{\alpha !} \int_{R^{n}} \frac{(x-y)^{\alpha} D^{\alpha} \tilde{b}(y)}{|x-y|^{m}} K(x, y) f_{1}(y) d y \\
& +\int_{R^{n}} \frac{R_{m+1}(\tilde{b} ; x, y)}{|x-y|^{m}} K(x, y) f_{2}(y) d y \\
= & T\left(\frac{R_{m}(\tilde{b} ; x, \cdot)}{|x-\cdot|^{m}} f_{1}\right)-T\left(\sum_{|\alpha|=m} \frac{1}{\alpha !} \frac{(x-\cdot)^{\alpha} D^{\alpha} \tilde{b}}{|x-\cdot|^{m}} f_{1}\right)+T^{\tilde{b}}\left(f_{2}\right)(x)
\end{aligned}
$$

and

$$
\begin{aligned}
A_{t_{Q}} T^{b}(f)(x)= & \int_{R^{n}} \frac{R_{m}\left(\tilde{b}_{j} ; x, y\right)}{|x-y|^{m}} K_{t}(x, y) f_{1}(y) d y \\
& -\sum_{|\alpha|=m} \frac{1}{\alpha !} \int_{R^{n}} \frac{(x-y)^{\alpha} D^{\alpha} \tilde{b}(y)}{|x-y|^{m}} K_{t}(x, y) f_{1}(y) d y \\
& +\int_{R^{n}} \frac{R_{m+1}(\tilde{b} ; x, y)}{|x-y|^{m}} K_{t}(x, y) f_{2}(y) d y \\
= & A_{t_{Q}} T\left(\frac{R_{m}(\tilde{b} ; x, \cdot)}{|x-\cdot|^{m}} f_{1}\right)-A_{t_{Q}} T\left(\sum_{|\alpha|=m} \frac{1}{\alpha !} \frac{(x-\cdot)^{\alpha} D^{\alpha} \tilde{b}}{|x-\cdot|^{m}} f_{1}\right)+A_{t_{Q}} T^{\tilde{b}}\left(f_{2}\right)(x),
\end{aligned}
$$


then

$$
\begin{aligned}
& \left(\frac{1}{|Q|} \int_{Q}\left|T^{b}(f)(x)-A_{t_{Q}} T^{b}(f)(x)\right|^{\eta} d x\right)^{1 / \eta} \\
& \leq C\left(\frac{1}{|Q|} \int_{Q}\left|T\left(\frac{R_{m}(\tilde{b} ; x, \cdot)}{|x-\cdot|^{m}} f_{1}\right)(x)\right|^{\eta} d x\right)^{1 / \eta} \\
& \quad+C\left(\frac{1}{|Q|} \int_{Q}\left|T\left(\sum_{|\alpha|=m} \frac{1}{\alpha !} \frac{(x-\cdot)^{\alpha} D^{\alpha} \tilde{b}}{|x-\cdot|^{m}} f_{1}\right)(x)\right|^{\eta} d x\right)^{1 / \eta} \\
& \quad+C\left(\frac{1}{|Q|} \int_{Q}\left|A_{t_{Q}} T\left(\frac{R_{m}(\tilde{b} ; x, \cdot)}{|x-\cdot|^{m}} f_{1}\right)(x)\right|^{\eta} d x\right)^{1 / \eta} \\
& \quad+C\left(\frac{1}{|Q|} \int_{Q}\left|A_{t_{Q}} T\left(\sum_{|\alpha|=m} \frac{1}{\alpha !} \frac{(x-\cdot)^{\alpha} D^{\alpha} \tilde{b}}{|x-\cdot|^{m}} f_{1}\right)(x)\right|^{\eta} d x\right)^{1 / \eta} \\
& \quad+C\left(\frac{1}{|Q|} \int_{Q}\left|T^{\tilde{b}}\left(f_{2}\right)(x)-A_{t_{Q}} T^{\tilde{b}}\left(f_{2}\right)(x)\right|^{\eta} d x\right)^{1 / \eta} \\
& =I_{1}+I_{2}+I_{3}+I_{4}+I_{5} .
\end{aligned}
$$

For $I_{1}$, noting that $w \in A_{1}, w$ satisfies the reverse of Hölder's inequality

$$
\left(\frac{1}{|Q|} \int_{Q} w(x)^{p_{0}} d x\right)^{1 / p_{0}} \leq \frac{C}{|Q|} \int_{Q} w(x) d x
$$

for all cube $Q$ and some $1<p_{0}<\infty$ (see [1]). We take $q=r p_{0} /\left(r+p_{0}-1\right)$ in Lemma 7 and have $1<q<r$ and $p_{0}=q(r-1) /(r-q)$, then by Lemma 7 and Hölder's inequality, we get

$$
\begin{aligned}
\left|R_{m}(\tilde{b} ; x, y)\right| \leq & C|x-y|^{m} \sum_{|\alpha|=m}\left(\frac{1}{|\tilde{Q}(x, y)|} \int_{\tilde{Q}(x, y)}\left|D^{\alpha} \tilde{b}(z)\right|^{q} d z\right)^{1 / q} \\
\leq & C|x-y|^{m} \sum_{|\alpha|=m}|\tilde{Q}|^{-1 / q}\left(\int_{\tilde{Q}(x, y)}\left|D^{\alpha} \tilde{b}(z)\right|^{q} w(z)^{q(1-r) / r} w(z)^{q(r-1) / r} d z\right)^{1 / q} \\
\leq & C|x-y|^{m} \sum_{|\alpha|=m}|\tilde{Q}|^{-1 / q}\left(\int_{\tilde{Q}(x, y)}\left|D^{\alpha} \tilde{b}(z)\right|^{r} w(z)^{1-r} d z\right)^{1 / r} \\
& \times\left(\int_{\tilde{Q}(x, y)} w(z)^{q(r-1) /(r-q)} d z\right)^{(r-q) / r q} \\
\leq & C|x-y|^{m} \sum_{|\alpha|=m}|\tilde{Q}|^{-1 / q}\left\|D^{\alpha} b\right\|_{B M O(w)} w(\tilde{Q})^{1 / r}|\tilde{Q}|^{(r-q) / r q} \\
& \times\left(\frac{1}{|\tilde{Q}(x, y)|} \int_{\tilde{Q}(x, y)} w(z)^{p_{0}} d z\right)^{(r-q) / r q} \\
\leq & C|x-y|^{m} \sum_{|\alpha|=m}\left\|D^{\alpha} b\right\|_{B M O(w)}|\tilde{Q}|^{-1 / q} w(\tilde{Q})^{1 / r}|\tilde{Q}|^{1 / q-1 / r} \\
& \times\left(\frac{1}{|\tilde{Q}(x, y)|} \int_{\tilde{Q}(x, y)} w(z) d z\right)^{(r-1) / r}
\end{aligned}
$$




$$
\begin{aligned}
& \leq C|x-y|^{m} \sum_{|\alpha|=m}\left\|D^{\alpha} b\right\|_{B M O(w)}|\tilde{Q}|^{-1 / q} w(\tilde{Q})^{1 / r}|\tilde{Q}|^{1 / q-1 / r} w(\tilde{Q})^{1-1 / r}|\tilde{Q}|^{1 / r-1} \\
& \leq C|x-y|^{m} \sum_{|\alpha|=m}\left\|D^{\alpha} b\right\|_{B M O(w)} \frac{w(\tilde{Q})}{|\tilde{Q}|} \\
& \leq C|x-y|^{m} \sum_{|\alpha|=m}\left\|D^{\alpha} b\right\|_{B M O(w)} w(\tilde{x}) .
\end{aligned}
$$

Thus, by the $L^{s}$-boundedness of $T$ (see Lemma 2) for $1<s<r$ and $w \in A_{1} \subseteq A_{r / s}$, we obtain

$$
\begin{aligned}
I_{1} \leq & \frac{C}{|Q|} \int_{Q}\left|T\left(\frac{R_{m}(\tilde{b} ; x, \cdot)}{|x-\cdot|^{m}} f_{1}\right)(x)\right| d x \\
\leq & C \sum_{|\alpha|=m}\left\|D^{\alpha} b\right\|_{B M O(w)} w(\tilde{x})\left(\frac{1}{|Q|} \int_{R^{n}}\left|T\left(f_{1}\right)(x)\right|^{s} d x\right)^{1 / s} \\
\leq & C \sum_{|\alpha|=m}\left\|D^{\alpha} b\right\|_{B M O(w)} w(\tilde{x})|Q|^{-1 / s}\left(\int_{R^{n}}\left|f_{1}(x)\right|^{s} d x\right)^{1 / s} \\
\leq & C \sum_{|\alpha|=m}\left\|D^{\alpha} b\right\|_{B M O(w)} w(\tilde{x})|Q|^{-1 / s}\left(\int_{\tilde{Q}}|f(x)|^{s} w(x)^{s / r} w(x)^{-s / r} d x\right)^{1 / s} \\
\leq & C \sum_{|\alpha|=m}\left\|D^{\alpha} b\right\|_{B M O(w)} w(\tilde{x})|Q|^{-1 / s}\left(\int_{\tilde{Q}}|f(x)|^{r} w(x) d x\right)^{1 / r}\left(\int_{\tilde{Q}} w(x)^{-s /(r-s)} d x\right)^{(r-s) / r s} \\
\leq & C \sum_{|\alpha|=m}\left\|D^{\alpha} b\right\|_{B M O(w)} w(\tilde{x})|Q|^{-1 / s} w(\tilde{Q})^{1 / r}\left(\frac{1}{w(\tilde{Q})} \int_{\tilde{Q}}|f(x)|^{r} w(x) d x\right)^{1 / r} \\
& \times\left(\frac{1}{|\tilde{Q}|} \int_{\tilde{Q}} w(x)^{-s /(r-s)} d x\right)^{(r-s) / r s}\left(\frac{1}{|\tilde{Q}|} \int_{\tilde{Q}} w(x) d x\right)^{1 / r}|\tilde{Q}|^{1 / s} w(\tilde{Q})^{-1 / r} \\
\leq & C \sum_{|\alpha|=m}\left\|D^{\alpha} b\right\|_{B M O(w)} w(\tilde{x}) M_{r, w}(f)(\tilde{x}) .
\end{aligned}
$$

For $I_{2}$, by the weak $\left(L^{1}, L^{1}\right)$ boundedness of $T$ (see Lemma 2) and Kolmogorov's inequality (see Lemma 1), we obtain

$$
\begin{aligned}
I_{2} & \leq C \sum_{|\alpha|=m}\left(\frac{1}{|Q|} \int_{Q}\left|T\left(D^{\alpha} \tilde{b} f_{1}\right)(x)\right|^{\eta} d x\right)^{1 / \eta} \\
& \leq C \sum_{|\alpha|=m} \frac{|Q|^{1 / \eta-1}}{|Q|^{1 / \eta}} \frac{\left\|T\left(D^{\alpha} \tilde{b} f_{1}\right) \chi_{Q}\right\|_{L^{\eta}}}{\left\|\chi_{Q}\right\|_{L^{\eta /(1-\eta)}}} \\
& \leq C \sum_{|\alpha|=m} \frac{1}{|Q|}\left\|T\left(D^{\alpha} \tilde{b} f_{1}\right)\right\|_{W L^{1}} \\
& \leq C \sum_{|\alpha|=m} \frac{1}{|Q|} \int_{R^{n}}\left|D^{\alpha} \tilde{b}(x) f_{1}(x)\right| d x \\
& \leq C \sum_{|\alpha|=m} \frac{1}{|Q|} \int_{\tilde{Q}}\left|D^{\alpha} b(x)-\left(D^{\alpha} b\right)_{\tilde{Q}}\right| w(x)^{-1 / r}|f(x)| w(x)^{1 / r} d x \\
& \leq C \sum_{|\alpha|=m} \frac{1}{|Q|}\left(\int_{\tilde{Q}}\left|\left(D^{\alpha} b(x)-\left(D^{\alpha} b\right)_{\tilde{Q}}\right)\right|^{r^{\prime}} w(x)^{1-r^{\prime}} d x\right)^{1 / r^{\prime}}\left(\int_{\tilde{Q}}|f(x)|^{r} w(x) d x\right)^{1 / r}
\end{aligned}
$$




$$
\begin{aligned}
& \leq C \sum_{|\alpha|=m} \frac{1}{|Q|}\left\|D^{\alpha} b\right\|_{B M O(w)} w(\tilde{Q})^{1 / r^{\prime}} w(\tilde{Q})^{1 / r}\left(\frac{1}{w(\tilde{Q})} \int_{\tilde{Q}}|f(x)|^{r} w(x) d x\right)^{1 / r} \\
& \leq C \sum_{|\alpha|=m}\left\|D^{\alpha} b\right\|_{B M O(w)} \frac{w(\tilde{Q})}{|\tilde{Q}|} M_{r, w}(f)(\tilde{x}) \\
& \leq C \sum_{|\alpha|=m}\left\|D^{\alpha} b\right\|_{B M O(w)} w(\tilde{x}) M_{r, w}(f)(\tilde{x}) .
\end{aligned}
$$

For $I_{3}$ and $I_{4}$, by Lemma 8 and similar to the proof of $I_{1}$ and $I_{2}$, we get

$$
\begin{aligned}
I_{3} & \leq \frac{C}{|Q|} \int_{Q}\left|T\left(\frac{R_{m}(\tilde{b} ; x, \cdot)}{|x-\cdot|^{m}} f_{1}\right)(x)\right| d x \\
& \leq C \sum_{|\alpha|=m}\left\|D^{\alpha} b\right\|_{B M O(w)} w(\tilde{x}) M_{r, w}(f)(\tilde{x}), \\
I_{4} & \leq C \sum_{|\alpha|=m}\left(\frac{1}{|Q|} \int_{Q}\left|T\left(D^{\alpha} \tilde{b} f_{1}\right)(x)\right|^{\eta} d x\right)^{1 / \eta} \\
& \leq C \sum_{|\alpha|=m}\left\|D^{\alpha} b\right\|_{B M O(w)} w(\tilde{x}) M_{r, w}(f)(\tilde{x}) .
\end{aligned}
$$

For $I_{5}$, note that $|x-y| \approx\left|x_{0}-y\right|$ for $x \in Q$ and $y \in R^{n} \backslash Q$. We have, by Lemma 7 and similar to the proof of $I_{1}$,

$$
\left|R_{m}(\tilde{b} ; x, y)\right| \leq C|x-y|^{m} \sum_{|\alpha|=m}\left\|D^{\alpha} b\right\|_{B M O(w)} w(\tilde{x}) .
$$

Thus, by the conditions on $K$ and $K_{t}$, and $w \in A_{1} \subseteq A_{r}$,

$$
\begin{aligned}
& \left|T^{\tilde{b}}\left(f_{2}\right)(x)-A_{t_{Q}} T^{\tilde{b}}\left(f_{2}\right)\left(x_{0}\right)\right| \\
& \leq \int_{R^{n}} \frac{\left|R_{m}(\tilde{b} ; x, y)\right|}{|x-y|^{m}}\left|K(x, y)-K_{t}(x, y)\right|\left|f_{2}(y)\right| d y \\
& \quad+\sum_{|\alpha|=m} \frac{1}{\alpha !} \int_{R^{n}} \frac{\left|D^{\alpha} \tilde{b}_{1}(y)\right|\left|(x-y)^{\alpha_{1}}\right|}{|x-y|^{m}}\left|K(x, y)-K_{t}(x, y)\right|\left|f_{2}(y)\right| d y \\
& \leq \sum_{k=0}^{\infty} \sum_{|\alpha|=m}\left\|D^{\alpha} b\right\|_{B M O(w)} w(\tilde{x}) \int_{2^{k+1} \tilde{Q} \backslash 2^{k} \tilde{Q}} \frac{d^{\delta}}{\left|x_{0}-y\right|^{n+\delta}}|f(y)| w(y)^{1 / r} w(y)^{-1 / r} d y \\
& \quad+C \sum_{|\alpha|=m} \sum_{k=0}^{\infty} \int_{2^{k+1} \tilde{Q}}\left|\left(D^{\alpha} b\right)_{2^{k+1} \tilde{Q}}-\left(D^{\alpha} b\right)_{\tilde{Q}}\right| \frac{d^{\delta}}{\left|x_{0}-y\right|^{n+\delta}}|f(y)| w(y)^{1 / r} w(y)^{-1 / r} d y \\
& \quad+C \sum_{|\alpha|=m} \sum_{k=0}^{\infty} \int_{2^{k+1} \tilde{Q}}\left|D^{\alpha} b(y)-\left(D^{\alpha} b\right)_{2^{k+1} \tilde{Q}} \frac{d^{\delta}}{\left|x_{0}-y\right|^{n+\delta}}\right| f(y) \mid w(y)^{1 / r} w(y)^{-1 / r} d y \\
& \leq C \sum_{|\alpha|=m}\left\|D^{\alpha} b\right\|_{B M O(w)} w(\tilde{x}) \sum_{k=1}^{\infty} k \frac{d^{\delta}}{\left(2^{k} d\right)^{n+\delta}}\left(\int_{2^{k} \tilde{Q}}|f(y)|^{r} w(y) d x\right)^{1 / r} \\
& \quad \times\left(\frac{1}{\left|2^{k} \tilde{Q}\right|} \int_{2^{k} \tilde{Q}} w(y)^{-1 /(r-1)} d y\right)^{(r-1) / r}\left(\frac{1}{\left|2^{k} \tilde{Q}\right|} \int_{2^{k} \tilde{Q}} w(y) d y\right)^{1 / r}\left|2^{k} \tilde{Q}\right| w\left(2^{k} \tilde{Q}\right)^{-1 / r}
\end{aligned}
$$


Lu Journal of Inequalities and Applications 2014, 2014:276

Page 12 of 18

http://www.journalofinequalitiesandapplications.com/content/2014/1/276

$$
\begin{aligned}
& +C \sum_{|\alpha|=m} \sum_{k=1}^{\infty} \frac{d^{\delta}}{\left(2^{k} d\right)^{n+\delta}}\left(\int_{2^{k} \tilde{Q}}\left|D^{\alpha} b(y)-\left(D^{\alpha} b\right)_{2^{k} \tilde{Q}}\right|^{r^{\prime}} w(y)^{1-r^{\prime}} d y\right)^{1 / r^{\prime}} \\
& \times\left(\int_{2^{k} \tilde{Q}}|f(y)|^{r} w(y) d y\right)^{1 / r} \\
\leq & C \sum_{|\alpha|=m}\left\|D^{\alpha} b\right\|_{B M O(w)} w(\tilde{x}) \sum_{k=1}^{\infty} k 2^{-k \delta}\left(\frac{1}{w\left(2^{k} \tilde{Q}\right)} \int_{2^{k} \tilde{Q}}|f(y)|^{r} w(y) d x\right)^{1 / r} \\
& +C \sum_{|\alpha|=m}\left\|D^{\alpha} b\right\|_{B M O(w)} \sum_{k=1}^{\infty} 2^{-k \delta} \frac{w\left(2^{k} \tilde{Q}\right)}{\left|2^{k} \tilde{Q}\right|}\left(\frac{1}{w\left(2^{k} \tilde{Q}\right)} \int_{2^{k} \tilde{Q}}|f(y)|^{r} w(y) d x\right)^{1 / r} \\
\leq & C \sum_{|\alpha|=m}\left\|D^{\alpha} b\right\|_{B M O(w)} w(\tilde{x}) M_{r, w}(f)(\tilde{x}) \sum_{k=1}^{\infty} k 2^{-k \delta} \\
\leq & C \sum_{|\alpha|=m}\left\|D^{\alpha} b\right\|_{B M O(w)} w(\tilde{x}) M_{r, w}(f)(\tilde{x}) .
\end{aligned}
$$

Thus

$$
I_{5} \leq C \sum_{|\alpha|=m}\left\|D^{\alpha} b\right\|_{B M O(w)} w(\tilde{x}) M_{r, w}(f)(\tilde{x})
$$

These complete the proof of Theorem 1.

Proof of Theorem 2 It suffices to prove for $f \in C_{0}^{\infty}\left(R^{n}\right)$ and some constant $C_{0}$ that the following inequality holds:

$$
\left(\frac{1}{|Q|} \int_{Q}\left|T^{b}(f)(x)-A_{t_{Q}}\left(T^{b}(f)\right)(x)\right|^{\eta} d x\right)^{1 / \eta} \leq C \sum_{|\alpha|=m}\left\|D^{\alpha} b\right\|_{L i p_{\beta}(w)} w(\tilde{x}) M_{\beta, r, w}(f)(\tilde{x}),
$$

where $t_{Q}=d^{2}$ and $d$ denotes the side length of $Q$. Fix a cube $Q=Q\left(x_{0}, d\right)$ and $\tilde{x} \in Q$. Similar to the proof of Theorem 1 , we have, for $f_{1}=f \chi_{\tilde{Q}}$ and $f_{2}=f \chi_{R^{n} \backslash \tilde{Q}}$,

$$
\begin{aligned}
\left(\frac{1}{|Q|} \int_{Q}\left|T^{b}(f)(x)-A_{t_{Q}} T^{b}(f)(x)\right|^{\eta} d x\right)^{1 / \eta} & \\
\leq & \left(\frac{1}{|Q|} \int_{Q}\left|T\left(\frac{R_{m}(\tilde{b} ; x, \cdot)}{|x-\cdot|^{m}} f_{1}\right)(x)\right|^{\eta} d x\right)^{1 / \eta} \\
& +\left(\frac{1}{|Q|} \int_{Q}\left|T\left(\sum_{|\alpha|=m} \frac{1}{\alpha !} \frac{(x-\cdot)^{\alpha} D^{\alpha} \tilde{b}}{|x-\cdot|^{m}} f_{1}\right)(x)\right|^{\eta} d x\right)^{1 / \eta} \\
& +\left(\frac{1}{|Q|} \int_{Q}\left|A_{t_{Q}} T\left(\frac{R_{m}(\tilde{b} ; x, \cdot)}{|x-\cdot|^{m}} f_{1}\right)(x)\right|^{\eta} d x\right)^{1 / \eta} \\
& +\left(\frac{1}{|Q|} \int_{Q} \mid A_{t_{Q}} T\left(\sum_{|\alpha|=m} \frac{1}{\alpha !} \frac{(x-\cdot)^{\alpha} D^{\alpha} \tilde{b}}{|x-\cdot|^{m}} f_{1}\right)^{\left.\left.(x)\right|^{\eta} d x\right)^{1 / \eta}}\right. \\
& +\left(\frac{1}{|Q|} \int_{Q}\left|T^{\tilde{b}}\left(f_{2}\right)(x)-A_{t_{Q}} T^{\tilde{b}}\left(f_{2}\right)(x)\right|^{\eta} d x\right)^{1 / \eta} \\
= & +J_{2}+J_{3}+J_{4}+J_{5} .
\end{aligned}
$$


For $J_{1}$ and $J_{2}$, by using the same argument as in the proof of Theorem 1 , we get

$$
\begin{aligned}
& \left|R_{m}(\tilde{b} ; x, y)\right| \leq C|x-y|^{m} \sum_{|\alpha|=m}|\tilde{Q}|^{-1 / q}\left(\int_{\tilde{Q}(x, y)}\left|D^{\alpha} \tilde{b}(z)\right|^{q} w(z)^{q(1-r) / r} w(z)^{q(r-1) / r} d z\right)^{1 / q} \\
& \leq C|x-y|^{m} \sum_{|\alpha|=m}|\tilde{Q}|^{-1 / q}\left(\int_{\tilde{Q}(x, y)}\left|D^{\alpha} \tilde{b}(z)\right|^{r} w(z)^{1-r} d z\right)^{1 / r} \\
& \times\left(\int_{\tilde{Q}(x, y)} w(z)^{q(r-1) /(r-q)} d z\right)^{(r-q) / r q} \\
& \leq C|x-y|^{m} \sum_{|\alpha|=m}|\tilde{Q}|^{-1 / q}\left\|D^{\alpha} b\right\|_{L_{p_{\beta}}(w)} w(\tilde{Q})^{\beta / n+1 / r}|\tilde{Q}|^{(r-q) / r q} \\
& \times\left(\frac{1}{|\tilde{Q}(x, y)|} \int_{\tilde{Q}(x, y)} w(z)^{p_{0}} d z\right)^{(r-q) / r q} \\
& \leq C|x-y|^{m} \sum_{|\alpha|=m}\left\|D^{\alpha} b\right\|_{\operatorname{Lip}_{\beta}(w)}|\tilde{Q}|^{-1 / q} w(\tilde{Q})^{\beta / n+1 / r}|\tilde{Q}|^{1 / q-1 / r} \\
& \times\left(\frac{1}{|\tilde{Q}(x, y)|} \int_{\tilde{Q}(x, y)} w(z) d z\right)^{(r-1) / r} \\
& \leq C|x-y|^{m} \sum_{|\alpha|=m}\left\|D^{\alpha} b\right\|_{L_{p_{\beta}}(w)}|\tilde{Q}|^{-1 / q} w(\tilde{Q})^{\beta / n+1 / r}|\tilde{Q}|^{1 / q-1 / r} w(\tilde{Q})^{1-1 / r}|\tilde{Q}|^{1 / r-1} \\
& \leq C|x-y|^{m} \sum_{|\alpha|=m}\left\|D^{\alpha} b\right\|_{\operatorname{Lip}_{\beta}(w)} \frac{w(\tilde{Q})^{\beta / n+1}}{|\tilde{Q}|}
\end{aligned}
$$

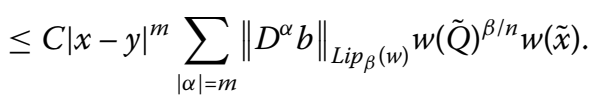

Thus

$$
\begin{aligned}
J_{1} \leq & C \sum_{|\alpha|=m}\left\|D^{\alpha} b\right\|_{L i p_{\beta}(w)} w(\tilde{Q})^{\beta / n} w(\tilde{x})|Q|^{-1 / s}\left(\int_{R^{n}}\left|f_{1}(x)\right|^{s} d x\right)^{1 / s} \\
\leq & C \sum_{|\alpha|=m}\left\|D^{\alpha} b\right\|_{L i p_{\beta}(w)} w(\tilde{Q})^{\beta / n} w(\tilde{x})|Q|^{-1 / s}\left(\int_{\tilde{Q}}|f(x)|^{r} w(x) d x\right)^{1 / r} \\
& \times\left(\int_{\tilde{Q}} w(x)^{-s /(r-s)} d x\right)^{(r-s) / r s} \\
\leq & C \sum_{|\alpha|=m}\left\|D^{\alpha} b\right\|_{L i p_{\beta}(w)} w(\tilde{x})|\tilde{Q}|^{-1 / s} w(\tilde{Q})^{1 / r}\left(\frac{1}{w(\tilde{Q})^{1-r \beta / n}} \int_{\tilde{Q}}|f(x)|^{r} w(x) d x\right)^{1 / r} \\
& \times\left(\frac{1}{|\tilde{Q}|} \int_{\tilde{Q}} w(x)^{-s /(r-s)} d x\right)^{(r-s) / r s}\left(\frac{1}{|\tilde{Q}|} \int_{\tilde{Q}} w(x) d x\right)^{1 / r}|\tilde{Q}|^{1 / s} w(\tilde{Q})^{-1 / r} \\
\leq & C \sum_{|\alpha|=m}\left\|D^{\alpha} b\right\|_{L i p_{\beta}(w)} w(\tilde{x}) M_{\beta, r, w}(f)(\tilde{x}), \\
J_{2} \leq & C \sum_{|\alpha|=m} \frac{1}{|Q|} \int_{\tilde{Q}}\left|D^{\alpha} b(x)-\left(D^{\alpha} b\right)_{\tilde{Q}}\right| w(x)^{-1 / r}|f(x)| w(x)^{1 / r} d x \\
\leq & C \sum_{|\alpha|=m} \frac{1}{|Q|}\left(\int_{\tilde{Q}} \mid\left(D^{\alpha} b(x)-\left.\left(D^{\alpha} b\right)_{\tilde{Q}}\right|^{r^{\prime}} w(x)^{1-r^{\prime}} d x\right)^{1 / r^{\prime}}\left(\int_{\tilde{Q}}|f(x)|^{r} w(x) d x\right)^{1 / r}\right.
\end{aligned}
$$




$$
\begin{aligned}
& \leq C \sum_{|\alpha|=m} \frac{1}{|Q|}\left\|D^{\alpha} b\right\|_{L_{p_{\beta}(w)} w(\tilde{Q})^{\beta / n+1 / r^{\prime}} w(\tilde{Q})^{1 / r-\beta / n}}\left(\frac{1}{w(\tilde{Q})^{1-r \beta / n}} \int_{\tilde{Q}}|f(x)|^{r} w(x) d x\right)^{1 / r} \\
& \leq C \sum_{|\alpha|=m}\left\|D^{\alpha} b\right\|_{L i p_{\beta}(w)} \frac{w(\tilde{Q})}{|\tilde{Q}|} M_{\beta, r, w}(f)(\tilde{x}) \\
& \leq C \sum_{|\alpha|=m}\left\|D^{\alpha} b\right\|_{L_{i p_{\beta}(w)}} w(\tilde{x}) M_{\beta, r, w}(f)(\tilde{x}) .
\end{aligned}
$$

For $J_{3}$ and $J_{4}$, by Lemma 9 and similar to the proof of $J_{1}$ and $J_{2}$, we get

$$
\begin{aligned}
J_{3} & \leq \frac{C}{|Q|} \int_{Q}\left|T\left(\frac{R_{m}(\tilde{b} ; x, \cdot)}{|x-\cdot|^{m}} f_{1}\right)(x)\right| d x \\
& \leq C \sum_{|\alpha|=m}\left\|D^{\alpha} b\right\|_{L i p_{\beta}(w)} w(\tilde{x}) M_{\beta, r, w}(f)(\tilde{x}), \\
J_{4} & \leq C \sum_{|\alpha|=m}\left(\frac{1}{|Q|} \int_{Q}\left|T\left(D^{\alpha} \tilde{b} f_{1}\right)(x)\right|^{\eta} d x\right)^{1 / \eta} \\
& \leq C \sum_{|\alpha|=m}\left\|D^{\alpha} b\right\|_{L i_{\beta}(w)} w(\tilde{x}) M_{\beta, r, w}(f)(\tilde{x}) .
\end{aligned}
$$

For $J_{5}$, by Lemma 7 and similar to the proof of $J_{1}$, for $k \geq 0$, we have

$$
\left|R_{m}(\tilde{b} ; x, y)\right| \leq C|x-y|^{m} \sum_{|\alpha|=m}\left\|D^{\alpha} b\right\|_{L i p_{\beta}(w)} w\left(2^{k} \tilde{Q}\right)^{\beta / n} w(\tilde{x}) .
$$

Thus

$$
\begin{aligned}
& \left|T^{\tilde{b}}\left(f_{2}\right)(x)-A_{t_{Q}} T^{\tilde{b}}\left(f_{2}\right)\left(x_{0}\right)\right| \\
& \leq \int_{R^{n}} \frac{\left|R_{m}(\tilde{b} ; x, y)\right|}{|x-y|^{m}}\left|K(x, y)-K_{t}(x, y)\right|\left|f_{2}(y)\right| d y \\
& \quad+\sum_{|\alpha|=m} \frac{1}{\alpha !} \int_{R^{n}} \frac{\left|D^{\alpha} \tilde{b}_{1}(y)\right| \mid(x-y)^{\alpha_{1} \mid}}{|x-y|^{m}}\left|K(x, y)-K_{t}(x, y)\right|\left|f_{2}(y)\right| d y \\
& \leq \sum_{k=0}^{\infty} \sum_{|\alpha|=m}\left\|D^{\alpha} b\right\|_{L i p_{\beta}(w)} w(\tilde{x}) w\left(2^{k} \tilde{Q}\right)^{\beta / n} \\
& \quad \times \int_{2^{k+1} \tilde{Q} \backslash 2^{k} \tilde{Q}} \frac{d^{\delta}}{\left|x_{0}-y\right|^{n+\delta}}|f(y)| w(y)^{1 / r} w(y)^{-1 / r} d y \\
& \quad+C \sum_{|\alpha|=m} \sum_{k=0}^{\infty} \int_{2^{k+1} \tilde{Q}}\left|\left(D^{\alpha} b\right)_{2^{k+1} \tilde{Q}}-\left(D^{\alpha} b\right)_{\tilde{Q}}\right| \frac{d^{\delta}}{\left|x_{0}-y\right|^{n+\delta}}|f(y)| w(y)^{1 / r} w(y)^{-1 / r} d y \\
& \quad+C \sum_{|\alpha|=m} \sum_{k=0}^{\infty} \int_{2^{k+1} \tilde{Q}}\left|D^{\alpha} b(y)-\left(D^{\alpha} b\right)_{2^{k+1} \tilde{Q}}\right| \frac{d^{\delta}}{\left|x_{0}-y\right|^{n+\delta}}|f(y)| w(y)^{1 / r} w(y)^{-1 / r} d y \\
& \leq C \sum_{|\alpha|=m}\left\|D^{\alpha} b\right\|_{L_{i p}(w)} w(\tilde{x}) \sum_{k=1}^{\infty} k \frac{d^{\delta}}{\left(2^{k} d\right)^{n+\delta}} w\left(2^{k} \tilde{Q}\right)^{\beta / n}\left(\int_{2^{k} \tilde{Q}}|f(y)|^{r} w(y) d x\right)^{1 / r} \\
& \quad \times\left(\frac{1}{\left|2^{k} \tilde{Q}\right|} \int_{2^{k} \tilde{Q}} w(y)^{-1 /(r-1)} d y\right)^{(r-1) / r}\left(\frac{1}{\left|2^{k} \tilde{Q}\right|} \int_{2^{k} \tilde{Q}} w(y) d y\right)^{1 / r}\left|2^{k} \tilde{Q}\right| w\left(2^{k} \tilde{Q}\right)^{-1 / r}
\end{aligned}
$$




$$
\begin{aligned}
& \quad+C \sum_{|\alpha|=m} \sum_{k=1}^{\infty} \frac{d^{\delta}}{\left(2^{k} d\right)^{n+\delta}}\left(\int_{2^{k} \tilde{Q}}\left|D^{\alpha} b(y)-\left(D^{\alpha} b\right)_{2^{k} \tilde{Q}}\right|^{r^{\prime}} w(y)^{1-r^{\prime}} d y\right)^{1 / r^{\prime}} \\
& \quad \times\left(\int_{2^{k} \tilde{Q}}|f(y)|^{r} w(y) d y\right)^{1 / r} \\
& \leq C \sum_{|\alpha|=m}\left\|D^{\alpha} b\right\|_{L i p_{\beta}(w)} w(\tilde{x}) \sum_{k=1}^{\infty} k 2^{-k \delta}\left(\frac{1}{w\left(2^{k} \tilde{Q}\right)^{1-r \beta / n}} \int_{2^{k} \tilde{Q}}|f(y)|^{r} w(y) d x\right)^{1 / r} \\
& \quad+C \sum_{|\alpha|=m}\left\|D^{\alpha} b\right\|_{L_{i p_{\beta}(w)}} \sum_{k=1}^{\infty} 2^{-k \delta} \frac{w\left(2^{k} \tilde{Q}\right)}{\left|2^{k} \tilde{Q}\right|}\left(\frac{1}{w\left(2^{k} \tilde{Q}\right)^{1-r \beta / n}} \int_{2^{k} \tilde{Q}}|f(y)|^{r} w(y) d x\right)^{1 / r} \\
& \leq C \sum_{|\alpha|=m}\left\|D^{\alpha} b\right\|_{L_{i p_{\beta}(w)} w(\tilde{x}) M_{\beta, r, w}(f)(\tilde{x}) .}
\end{aligned}
$$

This completes the proof of Theorem 2 .

Proof of Theorem 3 Choose $1<r<p$ in Theorem 1 and notice $w^{1-p} \in A_{1}$, then we have, by Lemmas 3 and 4 ,

$$
\begin{aligned}
\left\|T^{b}(f)\right\|_{L^{p}\left(w^{1-p}\right)} & \leq\left\|M_{\eta}\left(T^{b}(f)\right)\right\|_{L^{p}\left(w^{1-p}\right)} \leq C\left\|M_{A, \eta}^{\#}\left(T^{b}(f)\right)\right\|_{L^{p}\left(w^{1-p}\right)} \\
& \leq C \sum_{|\alpha|=m}\left\|D^{\alpha} b\right\|_{B M O(w)}\left\|w M_{r, w}(f)\right\|_{L^{p}\left(w^{1-p}\right)} \\
& =C \sum_{|\alpha|=m}\left\|D^{\alpha} b\right\|_{B M O(w)}\left\|M_{r, w}(f)\right\|_{L^{p}(w)} \\
& \leq C \sum_{|\alpha|=m}\left\|D^{\alpha} b\right\|_{B M O(w)}\|f\|_{L^{p}(w)} .
\end{aligned}
$$

This completes the proof of Theorem 3.

Proof of Theorem 4 Choose $1<r<p$ in Theorem 1 and notice $w^{1-p} \in A_{1}$, then we have, by Lemmas 5 and 6 ,

$$
\begin{aligned}
\left\|T^{b}(f)\right\|_{L^{p, \varphi}\left(w^{1-p}\right)} & \leq\left\|M_{\eta}\left(T^{b}(f)\right)\right\|_{L^{p, \varphi}\left(w^{1-p}\right)} \leq C\left\|M_{A, \eta}^{\#}\left(T^{b}(f)\right)\right\|_{L^{p, \varphi}\left(w^{1-p}\right)} \\
& \leq C \sum_{|\alpha|=m}\left\|D^{\alpha} b\right\|_{B M O(w)}\left\|w M_{r, w}(f)\right\|_{L^{p, \varphi}\left(w^{1-p}\right)} \\
& =C \sum_{|\alpha|=m}\left\|D^{\alpha} b\right\|_{B M O(w)}\left\|M_{r, w}(f)\right\|_{L^{p, \varphi}(w)} \\
& \leq C \sum_{|\alpha|=m}\left\|D^{\alpha} b\right\|_{B M O(w)}\|f\|_{L^{p, \varphi}(w)} .
\end{aligned}
$$

This completes the proof of Theorem 4 .

Proof of Theorem 5 Choose $1<r<p$ in Theorem 2 and notice $w^{1-q} \in A_{1}$, then we have, by Lemmas 3 and 4 ,

$$
\begin{aligned}
\left\|T^{b}(f)\right\|_{L^{q}\left(w^{1-q}\right)} & \leq\left\|M_{\eta}\left(T^{b}(f)\right)\right\|_{L^{q}\left(w^{1-q}\right)} \leq C\left\|M_{A, \eta}^{\#}\left(T^{b}(f)\right)\right\|_{L^{q}\left(w^{1-q}\right)} \\
& \leq C \sum_{|\alpha|=m}\left\|D^{\alpha} b\right\|_{L i p_{\beta}(w)}\left\|w M_{\beta, r, w}(f)\right\|_{L^{q}\left(w^{1-q}\right)}
\end{aligned}
$$




$$
\begin{aligned}
& =C \sum_{|\alpha|=m}\left\|D^{\alpha} b\right\|_{L_{L i p_{\beta}(w)}\left\|M_{\beta, r, w}(f)\right\|_{L^{q}(w)}} \\
& \leq C \sum_{|\alpha|=m}\left\|D^{\alpha} b\right\|_{L i p_{\beta}(w)}\|f\|_{L^{p}(w) .}
\end{aligned}
$$

This completes the proof of Theorem 5 .

Proof of Theorem 6 Choose $1<r<p$ in Theorem 2 and notice $w^{1-q} \in A_{1}$, then we have, by Lemmas 5 and 6 ,

$$
\begin{aligned}
\left\|T^{b}(f)\right\|_{L^{q, \varphi}\left(w^{1-q)}\right)} & \leq\left\|M_{\eta}\left(T^{b}(f)\right)\right\|_{L^{q, \varphi}\left(w^{1-q)}\right.} \leq C\left\|M_{A, \eta}^{\#}\left(T^{b}(f)\right)\right\|_{L^{q, \varphi}\left(w^{1-q}\right)} \\
& \leq C \sum_{|\alpha|=m}\left\|D^{\alpha} b\right\|_{L i p_{\beta}(w)}\left\|w M_{\beta, r, w}(f)\right\|_{L^{q, \varphi}\left(w^{1-q}\right)} \\
& =C \sum_{|\alpha|=m}\left\|D^{\alpha} b\right\|_{L i p_{\beta}(w)}\left\|M_{\beta, r, w}(f)\right\|_{L^{q, \varphi}(w)} \\
& \leq C \sum_{|\alpha|=m}\left\|D^{\alpha} b\right\|_{L i p_{\beta}(w)}\|f\|_{L^{p, \beta, \varphi}(w)} .
\end{aligned}
$$

This completes the proof of Theorem 6 .

\section{Applications}

In this section we shall apply the theorems of the paper to the holomorphic functional calculus of linear elliptic operators. First, we review some definitions regarding the holomorphic functional calculus (see [13]). Given $0 \leq \theta<\pi$. Define

$$
S_{\theta}=\{z \in C:|\arg (z)| \leq \theta\} \cup\{0\}
$$

and its interior by $S_{\theta}^{0}$. Set $\tilde{S}_{\theta}=S_{\theta} \backslash\{0\}$. A closed operator $L$ on some Banach space $E$ is said to be of type $\theta$ if its spectrum $\sigma(L) \subset S_{\theta}$ and for every $v \in(\theta, \pi]$, there exists a constant $C_{v}$ such that

$$
|\eta|\left\|(\eta I-L)^{-1}\right\| \leq C_{v}, \quad \eta \notin \tilde{S}_{\theta} .
$$

For $v \in(0, \pi]$, let

$$
H_{\infty}\left(S_{\mu}^{0}\right)=\left\{f: S_{\theta}^{0} \rightarrow C: f \text { is holomorphic and }\|f\|_{L^{\infty}}<\infty\right\},
$$

where $\|f\|_{L^{\infty}}=\sup \left\{|f(z)|: z \in S_{\mu}^{0}\right\}$. Set

$$
\Psi\left(S_{\mu}^{0}\right)=\left\{g \in H_{\infty}\left(S_{\mu}^{0}\right): \exists s>0, \exists c>0 \text { such that }|g(z)| \leq c \frac{|z|^{s}}{1+|z|^{2 s}}\right\} .
$$

If $L$ is of type $\theta$ and $g \in H_{\infty}\left(S_{\mu}^{0}\right)$, we define $g(L) \in L(E)$ by

$$
g(L)=-(2 \pi i)^{-1} \int_{\Gamma}(\eta I-L)^{-1} g(\eta) d \eta,
$$


where $\Gamma$ is the contour $\left\{\xi=r e^{ \pm i \phi}: r \geq 0\right\}$ parameterized clockwise around $S_{\theta}$ with $\theta<\phi<\mu$. If, in addition, $L$ is one-to-one and has a dense range, then, for $f \in H_{\infty}\left(S_{\mu}^{0}\right)$,

$$
f(L)=[h(L)]^{-1}(f h)(L),
$$

where $h(z)=z(1+z)^{-2}$. $L$ is said to have a bounded holomorphic functional calculus on the sector $S_{\mu}$ if

$$
\|g(L)\| \leq N\|g\|_{L^{\infty}}
$$

for some $N>0$ and for all $g \in H_{\infty}\left(S_{\mu}^{0}\right)$.

Now, let $L$ be a linear operator on $L^{2}\left(R^{n}\right)$ with $\theta<\pi / 2$ so that $(-L)$ generates a holomorphic semigroup $e^{-z L}, 0 \leq|\arg (z)|<\pi / 2-\theta$. Applying Theorem 6 of [12] and Theorems 1-6, we get the following.

\section{Corollary Assume that the following conditions are satisfied:}

(i) The holomorphic semigroup $e^{-z L}, 0 \leq|\arg (z)|<\pi / 2-\theta$ is represented by the kernels $a_{z}(x, y)$ which satisfy, for all $v>\theta$, an upper bound

$$
\left|a_{z}(x, y)\right| \leq c_{\nu} h_{|z|}(x, y)
$$

for $x, y \in R^{n}$, and $0 \leq|\arg (z)|<\pi / 2-\theta$, where $h_{t}(x, y)=C t^{-n / 2} s\left(|x-y|^{2} / t\right)$ and $s$ is a positive, bounded and decreasing function satisfying

$$
\lim _{r \rightarrow \infty} r^{n+\epsilon} s\left(r^{2}\right)=0
$$

(ii) The operator $L$ has a bounded holomorphic functional calculus in $L^{2}\left(R^{n}\right)$; that is, for all $v>\theta$ and $g \in H_{\infty}\left(S_{\mu}^{0}\right)$, the operator $g(L)$ satisfies

$$
\|g(L)(f)\|_{L^{2}} \leq c_{v}\|g\|_{L^{\infty}}\|f\|_{L^{2}}
$$

Then Theorems 1-6 hold for the multilinear operator $g(L)^{b}$ associated to $g(L)$ and $b$.

\section{Competing interests}

The author declares that he has no competing interests.

\section{Author's contributions}

The author completed the paper, and read and approved the final manuscript.

\section{Acknowledgements}

Project was supported by Scientific Research Fund of Hunan Provincial Education Departments (13C1007).

Received: 18 January 2014 Accepted: 24 May 2014 Published: 24 July 2014

\section{References}

1. Garcia-Cuerva, J, Rubio de Francia, JL: Weighted Norm Inequalities and Related Topics. North-Holland Mathematics Studies, vol. 16. North-Holland, Amsterdam (1985)

2. Stein, EM: Harmonic Analysis: Real Variable Methods, Orthogonality and Oscillatory Integrals. Princeton University Press, Princeton (1993)

3. Coifman, RR, Rochberg, R, Weiss, G: Factorization theorems for Hardy spaces in several variables. Ann. Math. 103, 611-635 (1976)

4. Pérez, C: Endpoint estimate for commutators of singular integral operators. J. Funct. Anal. 128, 163-185 (1995) 
5. Pérez, C, Trujillo-Gonzalez, R: Sharp weighted estimates for multilinear commutators. J. Lond. Math. Soc. 65, 672-692 (2002)

6. Chanillo, S: A note on commutators. Indiana Univ. Math. J. 31, 7-16 (1982)

7. Janson, S: Mean oscillation and commutators of singular integral operators. Ark. Mat. 16, 263-270 (1978)

8. Paluszynski, M: Characterization of the Besov spaces via the commutator operator of Coifman, Rochberg and Weiss. Indiana Univ. Math. J. 44, 1-17 (1995)

9. Bloom, S: A commutator theorem and weighted BMO. Trans. Am. Math. Soc. 292, 103-122 (1985)

10. $\mathrm{Hu}, \mathrm{B}, \mathrm{Gu}, \mathrm{J}$ : Necessary and sufficient conditions for boundedness of some commutators with weighted Lipschitz spaces. J. Math. Anal. Appl. 340, 598-605 (2008)

11. He, YX, Wang, YS: Commutators of Marcinkiewicz integrals and weighted BMO. Acta Math. Sin. Chin. Ser. 54, 513-520 (2011)

12. Duong, XT, Mclntosh, A: Singular integral operators with non-smooth kernels on irregular domains. Rev. Mat. Iberoam. 15, 233-265 (1999)

13. Martell, JM: Sharp maximal functions associated with approximations of the identity in spaces of homogeneous type and applications. Stud. Math. 161, 113-145 (2004)

14. Deng, DG, Yan, LX: Commutators of singular integral operators with non-smooth kernels. Acta Math. Sci. 25, 137-144 (2005)

15. Liu, LZ: Sharp function boundedness for vector-valued multilinear singular integral operators with non-smooth kernels. J. Contemp. Math. Anal. 45, 185-196 (2010)

16. Liu, LZ: Multilinear singular integral operators on Triebel-Lizorkin and Lebesgue spaces. Bull. Malays. Math. Sci. Soc. 35, 1075-1086 (2012)

17. Zhou, XS, Liu, LZ: Weighted boundedness for multilinear singular integral operators with non-smooth kernels on Morrey space. Rev. R. Acad. Cienc. Exactas Fís. Nat., Ser. A Mat. 104, 115-127 (2010)

18. Cohen, J, Gosselin, J: On multilinear singular integral operators on $R^{n}$. Stud. Math. 72, 199-223 (1982)

19. Cohen, J, Gosselin, J: A BMO estimate for multilinear singular integral operators. III. J. Math. 30, 445-465 (1986)

20. Ding, Y, Lu, SZ: Weighted boundedness for a class rough multilinear operators. Acta Math. Sin. 17, 517-526 (2001)

21. Garcia-Cuerva, J: Weighted $H^{P}$ Spaces. Dissert. Math., vol. 162 (1979)

22. Di Fazio, G, Ragusa, MA: Interior estimates in Morrey spaces for strong solutions to nondivergence form equations with discontinuous coefficients. J. Funct. Anal. 112, 241-256 (1993)

23. Peetre, J: On convolution operators leaving $L^{p, \lambda}$-spaces invariant. Ann. Mat. Pura Appl. 72, 295-304 (1966)

24. Peetre, J: On the theory of $L^{p, \lambda}$-spaces. J. Funct. Anal. 4, 71-87 (1969)

25. Di Fazio, G, Ragusa, MA: Commutators and Morrey spaces. Boll. Unione Mat. Ital., A 5, 323-332 (1991)

26. Liu, LZ: Interior estimates in Morrey spaces for solutions of elliptic equations and weighted boundedness for commutators of singular integral operators. Acta Math. Sci. Ser. B 25, 89-94 (2005)

27. Mizuhara, T: Boundedness of some classical operators on generalized Morrey spaces. In: Harmonic Analysis: Proceedings of a Conference Held in Sendai, Japan, pp. 183-189 (1990)

\section{Submit your manuscript to a SpringerOpen ${ }^{\circ}$ journal and benefit from:}

- Convenient online submission

Rigorous peer review

- Immediate publication on acceptance

- Open access: articles freely available online

- High visibility within the field

- Retaining the copyright to your article 